\title{
Seed island formation by forced magnetic reconnection
}

\author{
Q. Yu, S. Günter, K. Lackner, and M. Maraschek \\ Max-Planck-Institut für Plasmaphysik, EURATOM Association, 85748 Garching, Germany
}

Neoclassical tearing modes observed in experiments often grow from seed magnetic islands induced by triggers like sawteeth. The formation of seed islands is studied in this paper using both the reduced MHD and two-fluid equations, with the trigger being modelled by externally applied resonant magnetic perturbations. In the linear phase the growth rate of the driven mode is found to be the same as that of the trigger. A slowly growing trigger drives a tearing mode, while a fast one drives a kink-like mode, which becomes a tearing mode later when the trigger's growth slows down. A finite ion sound Larmor radius (ion Larmor radius by using electron temperature) and electron inertia are found to lead to a larger seed island for a given external perturbation. The electron diamagnetic drift and plasma rotation, if increasing the relative rotation between the trigger and the driven mode, decrease the seed island width. 


\section{Introduction}

It is well known that for a sufficiently high bootstrap current fraction, neoclassical tearing modes (NTMs) limit the plasma pressure or even cause disruptions in tokamak experiments, being an important issue for a fusion reactor [1-12]. NTMs observed in experiments often grow from small seed magnetic islands generated by triggers like sawteeth [3-12], as expected from theoretical predictions [13-24]. Small amplitude tearing modes are predicted to be stabilized by the electron diamagnetic drift and the ion polarization current in their linear and early nonlinear phases even for a positive $\Delta^{\prime}$, where $\Delta^{\prime}$ is the tearing mode stability index [1322]. In addition, the Glasser effect also provides a stabilizing effect $[5,23,24]$. When a sufficiently large seed island is driven by a trigger, it can be further destabilized by the perturbed bootstrap current to develop into a large amplitude NTM [1-12]. For the drift-tearing mode driven by the electron temperature gradient, a sufficiently large trigger is also found to drive a small amplitude mode into a NTM [25,26]. Due to nonlinear and toroidal effects, a seed island for a NTM can be generated by instabilities of different helicities. For example, an internal $\mathrm{m} / \mathrm{n}=1 / 1$ kink mode will drive non-linearly its second harmonic, the $2 / 2$ component, and also the $3 / 2$ component perturbation due to toroidicity in tokamak plasmas ( $\mathrm{m}$ and $\mathrm{n}$ are the poloidal and toroidal mode numbers respectively) [6,9]. Edge localized modes (ELMs), resistive wall modes or other instabilities could also act as triggers to drive seed islands $[5,8,10]$.

According to the modified Rutherford equation, the critical island width for the NTM's onset is described by the ion polarization current and the transport model, leading to a critical seed island width being close to the poloidal ion gyroradius, which is about a few centimetres for ASDEX Upgrade and DIII-D [5-11,15-20]. Because of its small size the seed island width cannot be directly measured in experiments. Theoretical investigation is required to look into 
the seed island physics, to understand existing experimental results and to provide predictions for ITER. The seed island formation can be regarded as a process of forced magnetic reconnection, a subject of concern in laboratory plasmas, magnetotail and coronal plasmas [2741], being similar to the penetration of resonant magnetic perturbations (RMPs) into a plasma [27-37]. The seed island growth was studied analytically earlier using the reduced MHD equations [27]. In the present paper both the reduced MHD and two-fluid equations are utilized for a more complete understanding of the seed island formation. The triggers are modelled by externally applied RMPs.

In Section 2 our theoretical model is described. The numerical results obtained from reduced MHD equations are presented in Section 3, and a corresponding heuristic linear analysis is included in the Appendix for understanding and comparison with numerical results, which reveals that the driven mode is of a kink-like type for a sufficiently fast growing trigger. The finite $\rho_{\mathrm{s}}$ (ion Larmor radius by using electron temperature) effect on the seed island growth is given in Section 4. In Section 5 the electron inertia effect and the relative rotation between the trigger and the driven mode, caused by the electron diamagnetic drift and by plasma rotation, are further taken into account with ASDEX Upgrade and reactor relevant parameters as input. Finally, the discussion and summary are presented in Section 6.

\section{Theoretical model}

The large aspect-ratio tokamak approximation is utilized. The magnetic field is defined as $\mathbf{B}=\mathrm{B}_{0 \mathrm{t}}\left(\mathbf{e}_{\mathrm{t}}-\mathbf{e}_{\theta} \mathrm{k}_{\mathrm{t}} / \mathrm{k}_{\theta}\right)+\nabla \psi \times \mathbf{e}_{t}$, where $\psi$ is the helical flux function, $\mathrm{k}_{\theta}=\mathrm{m} / \mathrm{r}$ and $\mathrm{k}_{\mathrm{t}}=\mathrm{n} / \mathrm{R}$ are the wave vector in $\mathbf{e}_{\theta}$ (poloidal) and $\mathbf{e}_{\mathrm{t}}$ (toroidal) direction, $\mathrm{r}$ and $\mathrm{R}$ are the minor and the major radius, and the subscript 0 denotes an equilibrium quantity. The plasma velocity is given by $\mathbf{v}=\mathrm{V}_{\|} \mathbf{e}_{\|}$ $+\nabla \phi \times \mathbf{e}_{t}$, where $\phi$ is the stream function.

The two-fluid equations utilized here include the mass conservation equation, the 
generalized Ohm's law, and the equation of motion in the perpendicular (after taking $\mathbf{e}_{t} \nabla \times$ ) and the parallel (to magnetic field) direction. Normalizing the length to the minor radius a, the time $t$ to the resistive time $\tau_{R}=a^{2} \mu_{0} / \eta_{0}$ ( $\eta_{0}$ is the characteristic resistivity), the helical flux $\psi$ to aB $B_{0 t}, \mathbf{v}$ to $a / \tau_{R}$, and the electron density $n_{e}$ to its value at the magnetic axis, these equations become [34]

$$
\begin{aligned}
& \frac{d n_{e}}{d t}=d_{1} \nabla_{\|} j-\nabla_{\|}\left(n_{e} v_{\|}\right)+\nabla_{\perp}\left(D_{\perp} \nabla_{\perp} n_{e}\right)+S_{n}, \\
& \frac{d \psi}{d t}=E_{0}-\eta j-\frac{\eta}{v_{e i}} \frac{d j}{d t}+\Omega \nabla_{\|} n_{e}, \\
& \frac{d U}{d t}=-S^{2} \nabla_{\|} j+\mu \nabla_{\perp}^{2} U+S_{m}, \\
& \frac{d v_{\|}}{d t}=-C_{s}^{2} \nabla_{\|} P / n_{e}+\mu \nabla_{\perp}^{2} v_{\|},
\end{aligned}
$$

where

$$
\begin{aligned}
& d / d t=\partial / \partial t+v_{\perp} \cdot \nabla_{\perp}, \\
& j=-\nabla_{\perp}^{2} \psi-2 n B_{0 t} /(m R)
\end{aligned}
$$

is the parallel plasma current density, and

$$
U=-\nabla_{\perp}^{2} \phi
$$

is the plasma vorticity. $\mathrm{S}=\tau_{\mathrm{R}} / \tau_{\mathrm{A}}, \tau_{\mathrm{A}}=\mathrm{a} / \mathrm{V}_{\mathrm{A}}$ is the toroidal Alfúen time, and $\eta$ is the normalized resistivity. $E_{0}$ is the equilibrium electric field, $S_{n}$ the particle source, and $S_{m}$ the poloidal momentum source leading to an equilibrium poloidal plasma rotation. $\mathrm{d}_{1}=\omega_{\mathrm{ce}} / \nu_{\mathrm{ei}}, \omega_{\mathrm{ce}}$ is the electron cyclotron frequency, $\Omega=\beta \mathrm{d}_{1}, \beta=4 \pi \mathrm{P} / \mathrm{B}_{0 \mathrm{t}}{ }^{2}$, and $\mathrm{P}=\mathrm{P}_{\mathrm{e}}$ is the electron pressure. $\mathrm{C}_{\mathrm{s}}, v_{\mathrm{ei}}$, $\mu$, and $\mathrm{D}_{\perp}$ are the normalized ion sound velocity, electron-ion collisional frequency, plasma viscosity, and perpendicular particle diffusivity. A constant electron temperature $T_{e}$ is assumed, and the cold ion assumption is made.

\section{Results obtained from reduced MHD equations}


Before presenting the results obtained from the two-fluid equations, it is helpful to first have a look at the seed island formation within the framework of the reduced MHD equations, which are obtained by neglecting the electron inertia and the density gradient in the generalized Ohm's law, the third and the fourth terms on the right hand side of equation (2). In this case only equations (2), (3) and (5)-(7) are solved by using the initial value code TM1, which has been used for modeling the nonlinear growth of drift-tearing modes and the plasma response to RMPs earlier $[25,33,34]$.

A monotonic profile for the safety factor $q$ is assumed with the $q=2$ surface located at $\mathrm{r}_{\mathrm{s}}=0.628 \mathrm{a}$, and the local magnetic shear length $\left.\mathrm{L}_{\mathrm{q}}=\mathrm{q} /(\mathrm{aq})^{\prime}\right)=0.336$. Only a single helicity, $\mathrm{m} / \mathrm{n}=2 / 1$, is considered. The plasma is stable against $\mathrm{m} / \mathrm{n}=2 / 1$ classical tearing modes if there is no externally applied trigger $\left(r_{s} \Delta^{\prime}=-1.2\right)$. The trigger for driving the seed island is taken into account by the following boundary condition,

$$
\psi_{a}=\psi_{a 0} \exp \left[\gamma_{f} t+i(m \theta+n \varphi)\right]
$$

for $\psi_{\mathrm{a}}<\psi_{\mathrm{a}, \max }$, and $\psi_{\mathrm{a}}$ is kept constant in time after $\psi_{\mathrm{a}}=\psi_{\mathrm{a}, \max }$ is reached, where $\psi_{\mathrm{a}}=\psi_{\mathrm{m} / \mathrm{n}, \mathrm{a}} / \mathrm{aB}$ ot is the normalized amplitude of the $\mathrm{m} / \mathrm{n}$ component of $\psi$ at $\mathrm{r}=\mathrm{a}, \gamma_{\mathrm{f}}$ is the growth rate of the trigger, and $\theta$ and $\varphi$ are the poloidal and toroidal angle. It is assumed to model growing ELMs or resistive wall modes as triggers. The boundary conditions for the stream function $\phi$ and the plasma vorticity $\mathrm{U}$ are $\phi=\mathrm{U}=0$ at $\mathrm{r}=\mathrm{a}$.

Figure 1 demonstrates the time evolution of the $\psi_{\mathrm{a}}$ with $\mathrm{m} / \mathrm{n}=2 / 1$ (dashed curve), which grows exponentially in time with $\gamma_{\mathrm{f}}=2 \times 10^{5} / \tau_{\mathrm{R}}$ from $\psi_{\mathrm{a} 0}=10^{-10}$ until $\psi_{\mathrm{a}, \max }=10^{-4}$ is reached at $\mathrm{t}=6.92 \times 10^{-5} \tau_{\mathrm{R}}$, and afterwards it is kept constant in time. The solid curve shows the induced $\mathrm{m} / \mathrm{n}=2 / 1$ component of the normalized helical flux, $\psi_{\mathrm{s}}=\psi_{2 / 1} / \mathrm{aB}_{0 \mathrm{t}}$, at the $\mathrm{q}=2$ surface, being much smaller than that at the plasma edge. $\psi_{\mathrm{s}}=1.34 \times 10^{-2} \psi_{\mathrm{a}}$ at $\mathrm{t}=6.92 \times 10^{-2} \tau_{\mathrm{R}}$. A small value of $\psi_{\mathrm{s}}$ is given at $\mathrm{t}=0$ in the calculation as the initial input. The dotted curve shows the normalized 
helical flux at the $\mathrm{q}=2$ surface using vacuum assumption. The following parameters, $\mathrm{S}=1.97 \times 10^{8}, \mu=0.21\left(\mathrm{a}^{2} / \tau_{\mathrm{R}}\right)$ and $\eta_{\mathrm{s}}=5.74$, have been used, where $\eta_{\mathrm{s}}$ is the normalized plasma resistivity at $\mathrm{q}=2$ surface. It will be seen from the following results that the input value of $\gamma_{\mathrm{f}}$ (and S) for figure 1 corresponds to the case that the driven mode is a tearing mode type. The duration of the growing phase of the trigger is determined by the values of $\gamma_{\mathrm{f}}, \psi_{a 0}$ and $\psi_{\mathrm{a} \text {,max }}$ defined by equation 8 . The value of $\psi_{\mathrm{a} 0}$ is chosen here such that it is sufficiently small, while the growing phase of the trigger is not too long.

Corresponding to figure 1 , the time evolution of the normalized growth rate of the driven mode, $\gamma \tau_{\mathrm{R}}$, calculated from $\left(\mathrm{d} \psi_{2 / 1} / \mathrm{dt}\right) / \psi_{2 / 1}$ at the $\mathrm{q}=2$ surface is shown in figure 2 by the solid curve. The dashed curve is the same as the solid one except for a smaller value of $\psi_{\mathrm{a} 0}\left(=10^{-14}\right)$ and $\psi_{s}(t=0)$, and these two curves overlap with each other from $t=0$ to $6.92 \times 10^{-5} \tau_{R}$. Three different phases are seen from figure 2:

(A) Initial phase: in which the value of $\gamma$ approaches $\gamma_{\mathrm{f}}$, as the initial input quantities for our numerical calculations are not eigenfunctions of the linear reduced MHD equations. The duration of this phase is in the order of $1 / \gamma_{\mathrm{f}}$ when a sufficiently small initial input $\psi_{\mathrm{s}}(\mathrm{t}=0)$ is utilized in calculations.

(B) Linear phase: in which the mode growth rate is the same as $\gamma_{\mathrm{f} .}$ The duration of this phase is determined by the initial RMP amplitude $\psi_{\mathrm{a} 0}$ for fixed values of $\gamma_{\mathrm{f}}$ and $\psi_{\mathrm{a} \text {,max }}$, as can be seen from the difference between the solid and dashed curves. The duration of the linear phase is longer for the dashed curve with $\psi_{\mathrm{a} 0}=10^{-14}$ than that for the solid one with $\psi_{\mathrm{a} 0}=10^{-10}$.

(C) Non-constant growth rate phase: in which the mode growth rate deviates from $\gamma_{\mathrm{f}}$. For the solid and dashed curves in figure 2 this phase begins when $\psi_{\mathrm{a}}$ reaches a constant, and afterwards $\gamma$ decreases in time. For a sufficiently large $\gamma_{\mathrm{f}}$ and $\psi_{\mathrm{a}, \max }$, however, this phase begins earlier before $\psi_{\mathrm{a}}$ reaches a constant due to nonlinear effect, as can be seen from the change of 
the radial location of the resonant surface to be shown in figure 7 .

Corresponding to figure 1 , the growth of the $m / n=2 / 1$ magnetic island is shown in figure 3 , where $r_{+}$is the island edge at $r>r_{s}$ at the helical angle passing through the island's o-point, and $r$ is the corresponding island edge at $\mathrm{r}<\mathrm{r}_{\mathrm{s}}$. The island width reaches about $0.01 \mathrm{a}$ at the end of the linear phase, as indicated by the vertical dashed line.

Figures 1-3 show an example for an originally stable $m / n=2 / 1$ mode driven to grow by the trigger. Depending on plasma parameters and the trigger's growth rate, in the linear phase the driven mode can be of either a tearing mode type or not. The local radial profiles of the normalized $\psi_{2 / 1}\left(\psi_{2 / 1} / \mathrm{aB}_{0 \mathrm{t}}\right)$ in the linear phase are shown in figure $4 \mathrm{a}$ for different values of $\mathrm{S}$ with $\gamma_{\mathrm{f}}=10^{6} / \tau_{\mathrm{R}}$. For a larger value of $\mathrm{S}$, corresponding to a smaller $\tau_{\mathrm{A}}$ in our calculations, $\psi_{2 / 1}$ is larger than zero across the resonant surface $\left(\mathrm{r}_{\mathrm{s}}=0.628 \mathrm{a}\right)$, being typical for a tearing mode. With decreasing $\mathrm{S}$ (increasing $\tau_{\mathrm{A}}$ ), $\psi_{2 / 1}$ becomes negative at $\mathrm{r}<\mathrm{r}_{\mathrm{s}}$, showing the feature of an ideal mode. Above results seems to be counter-intuitive. This is due to the normalization of the time to the resistive time $\tau_{R}$, so that the value of $\tau_{R}$ is fixed in our calculations, and a larger $S$ value corresponds to a smaller $\tau_{\mathrm{A}}$ rather than a larger $\tau_{\mathrm{R}}$. The results shown in figure $4 \mathrm{a}$ are expected from (A17)-(A20) in the Appendix, which reveal that a negative $\psi_{2 / 1}$ at $r<r_{s}$ corresponds to a driven kink mode, existing for a shorter growth time of the trigger compared to the local resistive diffusion time across the singular layer around the resonant surface. In the following the case with $\psi_{2 / 1}$ changing sign across the resonant surface will be called as the driven kink or kink-like mode due to the similarity between the wave-function given by (A19) and (A20) in the Appendix and those shown in reference 42 for the $m / n=1 / 1$ internal kink mode. While the case with $\psi_{2 / 1}$ having the same sign across the resonant surface will be called as driven tearing or tearing mode. For figure $4 \mathrm{a}$ the perturbed helical flux at the edge, $\psi_{\mathrm{a}}$, equals $2.2 \times 10^{-6}$, exceeding the $\psi_{2 / 1}$ at $q=2$ surface by two orders of magnitude for all four cases. 
Corresponding to figure $4 \mathrm{a}$, radial profiles of the normalized stream function, $\phi_{2 / 1} / \mathrm{a}^{2} \tau_{\mathrm{R}}$, are shown in figure $4 \mathrm{~b}$. For larger $\mathrm{S}$ values, $\phi_{2 / 1}$ changes sign across the resonant surface, being typical for a tearing mode. With decreasing value of $S, \phi_{2 / 1}$ approaches the typical behaviour of a kink mode. The corresponding plasma current density perturbation is found to have a broader radial profile for smaller values of S, being consistent with (A22) in the Appendix.

In figure 5 the boundary between the driven tearing and driven kink mode in their linear phase is shown in the $\mathrm{S}$ and $\gamma_{\mathrm{f}} \tau_{\mathrm{A}}$ plane by the dashed curve. The driven kink mode regime is found for $\tau_{\mathrm{A}} \gamma_{\mathrm{f}}>\mathrm{cS} \mathrm{S}_{\mathrm{s}}^{-1 / 3}$, being consistent with (A18) in the Appendix, where $\mathrm{S}_{\mathrm{s}}=\mathrm{S} \eta_{0} / \eta_{\mathrm{s}}$ is the value of $\mathrm{S}$ at $\mathrm{q}=2$ surface. From the dashed curve in figure 5 one finds $\mathrm{c}=1.02$ and $\mathrm{c}_{1}=\mathrm{c} /\left(\mathrm{k}_{\|}^{\prime}\right)^{2 / 3}=0.88$. The parameters $\mathrm{c}$ and $\mathrm{c}_{1}$ are defined in the Appendix.

\section{Effect of finite $\rho_{\mathrm{s}}$}

Finite $\rho_{\mathrm{s}}$ is known to speed up the forced magnetic reconnection, where $\rho_{\mathrm{s}}=\mathrm{T}_{\mathrm{e}} /\left(\mathrm{m}_{\mathrm{i}} \omega_{\mathrm{ci}}\right), \omega_{\mathrm{ci}}$ is the ion cyclotron frequency, and $m_{i}$ is the ion mass [38-41]. Following reference [38], the finite $\rho_{\mathrm{s}}$ effect is studied here at first by neglecting the equilibrium electron density gradient, the ion parallel velocity and the electron inertia in Equation (1)-(4). These effects will be included in the following Section. When the plasma viscosity and perpendicular particle diffusivity are assumed to be small, one finds from equations (1) and (3) that equation (2) becomes

$$
\frac{d \psi}{d t}=E_{0}-\eta j-\rho_{s n}^{2} \nabla_{\|} U
$$

where $\rho_{\mathrm{sn}}=\rho_{\mathrm{s}} / \mathrm{a}=\left(\Omega \mathrm{d}_{1}\right)^{1 / 2} / \mathrm{S}$.

To compare with results obtained from the reduced MHD equations $\left(\rho_{\mathrm{s}}=0\right)$, in figure 5 the boundary between the driven tearing and driven kink mode in the linear phase is shown in the $\mathrm{S}$ and $\gamma_{\mathrm{f}} \tau_{\mathrm{A}}$ plane for $\rho_{\mathrm{s}}=0.02 \mathrm{a}$ by the solid curve. The finite $\rho_{\mathrm{s}}$ effect extends the tearing mode regime to larger values of $\gamma_{\mathrm{f}}$. 
The local radial profile of the $\mathrm{m} / \mathrm{n}=2 / 1$ component of the normalized plasma current density in the linear phase is shown in figure 6a for $\rho_{\mathrm{s}} / \mathrm{a}=0.02,0.0067$ and 0 with $\gamma_{\mathrm{f}}=10^{6} / \tau_{\mathrm{R}}$, which is more peaked around the resonant surface for a larger $\rho_{\mathrm{s}}$, enhancing the magnetic reconnection as expected [38-41]. The corresponding radial profiles of the $\mathrm{m} / \mathrm{n}=2 / 1 \mathrm{component}$ of plasma vorticity, $U_{2 / 1}$, and stream function, $\phi_{2 / 1}$, are shown in figure $6 b$ and $6 c$, respectively.

The nonlinear growth of the $\mathrm{m} / \mathrm{n}=2 / 1$ island is shown in figure 7 with $\gamma_{\mathrm{f}} \tau_{\mathrm{R}}=10^{7}$ for $\rho_{\mathrm{s}}=0.02 \mathrm{a}$ (solid curve) and 0 (dotted curve). The vertical dashed line shows the time when $\psi_{\mathrm{a}}$ reaches $\psi_{\mathrm{a}, \max }=10^{-4}$. During the linear phase the modes are of the kink-like type for both cases due to the large value of $\gamma_{\mathrm{f}}$, as can be seen from Figure 5, and the mode growth rate is the same as $\gamma_{\mathrm{f}}$ in this phase, similar to that shown in figure 2. For the driven kink mode there is little magnetic reconnection, since $\psi_{2 / 1}$ is very small at the resonant surface and changes sign across it, similar to that shown in Figure 4a for lower $\mathrm{S}$ values. Therefore, the island width is quite small during the growing phase of the trigger. This is different from the case for a slowly growing trigger as shown in figures 1 and 3 with $\gamma_{\mathrm{f}} \tau_{\mathrm{R}}=2 \times 10^{5}$, for which the island width significantly increases during the linear phase and is much larger than that for figure 7 when $\psi_{\mathrm{a}}=\psi_{\mathrm{a} \text {, max }}=10^{-4}$ is reached, since the driven mode is of the tearing mode type for a low value of $\gamma_{\mathrm{f}}$. The outwards shift of the island edges from $\mathrm{t}=10^{-6} \tau_{\mathrm{R}}$ before $\psi_{\mathrm{a}}$ reaches a constant in figure 7 is due to the $\mathrm{m} / \mathrm{n}=0 / 0$ component of nonlinear plasma current density perturbation, which moves the $q=2$ surface slightly outwards. Significant magnetic reconnection begins when $\psi_{\mathrm{a}}$ reaches a constant, $\psi_{\mathrm{a}, \max }=10^{-4}$, as indicated by the vertical dashed line, and the mode changes from a driven kink into a driven tearing type. Afterwards the island width grows to about $0.01 \mathrm{a}$ in a time scale $10^{-}$ ${ }^{6} \tau_{\mathrm{R}}$, being faster for $\rho_{\mathrm{s}}=0.02 \mathrm{a}$ than that for $\rho_{\mathrm{s}}=0$. As expected, finite $\rho_{\mathrm{s}}$ effects are more significant for larger values of $\gamma_{\mathrm{f}}$.

For $\gamma_{\mathrm{f}} \tau_{\mathrm{R}}=10^{7}$, the nonlinear growth of the normalized $\mathrm{m} / \mathrm{n}=2 / 1$ island width, W/a, is shown 
in figure 8 for $\rho_{\mathrm{s}} / \mathrm{a}=0.02,0.01,0.0045$ and 0 . The vertical dashed line shows the time when $\psi_{\mathrm{a}}$ reaches a constant value, $\psi_{\mathrm{a}, \max }=10^{-4}$. Due to the large $\gamma_{\mathrm{f}}$ value utilized, the island begins its fast growth from that time and grows faster for a larger $\rho_{\mathrm{s}}$.

Compared to the mode driven by a trigger as discussed so far, the finite $\rho_{\mathrm{s}}$ effect on spontaneously growing tearing modes is quite different. This is shown in figure 9 , where the time evolution of the island width is given for $\rho_{\mathrm{s}} / \mathrm{a}=0.02,0.002,0.00067$ and 0 . For these calculations another equilibrium plasma current density profile is used for which the $\mathrm{m} / \mathrm{n}=2 / 1$ mode is unstable even for $\psi_{\mathrm{a}}=0$ due to a positive $\Delta^{\prime}$ value $\left(r_{s} \Delta^{\prime}=11\right)$. The faster island growth for a larger $\rho_{\mathrm{s}}$ here is due to a larger linear growth rate. Detailed numerical calculations for spontaneously growing tearing modes show that, if the value of $\rho_{\mathrm{s}}$ is sufficiently large, the dependence of the growth rate $\left(\sim \rho_{\mathrm{s}}^{2 / 3}\right)$ and linear layer width $\left(\sim \rho_{\mathrm{s}}^{-2 / 3}\right)$ on $\rho_{\mathrm{s}}$ agrees with that of the semi-collisional tearing mode theory in reference [41].

\section{Results for realistic plasma parameters}

NTMs triggered by sawtooth crashes and fishbone activity had been identified before from ASDEX Upgrade experimental data [7]. Efforts have also been devoted to identify if $\mathrm{m} / \mathrm{n}=2 / 1$ modes are triggered by ELMs. However, up to now only a few cases have been found on ASDEX Upgrade for which the 2/1 NTM onset might be correlated with ELMs.

To understand why 2/1 modes are not easily triggered by ELMs on ASDEX Upgrade, we further carry out our simulations based on typical ASDEX Upgrade experimental parameters except mentioned elsewhere. The toroidal magnetic field is $\mathrm{B}_{0 \mathrm{t}}=2 \mathrm{~T}$, and the plasma minor and major radius are $\mathrm{a}=0.5 \mathrm{~m}$ and $\mathrm{R}=1.7 \mathrm{~m}$, respectively. At the resonant surface the electron temperature $T_{e}=2 \mathrm{keV}$, and the electron density $\mathrm{n}_{\mathrm{e}}=3 \times 10^{19} \mathrm{~m}^{-3}$. The local electron diamagnetic drift frequency is about $f_{*}=-2 \mathrm{kHz}$. These parameters lead to $S_{\mathrm{s}}=2.65 \times 10^{8}, \Omega=9.43 \times 10^{4}$, 
$\mathrm{C}_{\mathrm{s}}=2.05 \times 10^{7}\left(\mathrm{a} / \tau_{\mathrm{R}}\right), \mathrm{d}_{1}=3.12 \times 10^{7}, v_{\mathrm{ei}}=2.2 \times 10^{4} / \mathrm{s}$, and $\rho_{\mathrm{s}} / \mathrm{a}=0.0065$. Assuming the perpendicular plasma viscosity to be at an anomalous transport level, e.g., $0.2 \mathrm{~m}^{2} / \mathrm{s}$, one finds $\mu=18.8\left(\mathrm{a}^{2} / \tau_{\mathrm{R}}\right)$ in normalized units. Furthermore, $D_{\perp}=\mu / 5$ is taken.

It is observed from in-vessel magnetic probes on ASDEX Upgrade that type-I ELMs typically grow in a time scale about $50 \mu \mathrm{s}$ and last for about $2 \mathrm{~ms}$ [43]. The amplitude of the resulting $\mathrm{m} / \mathrm{n}=2 / 1$ component perturbation is estimated to be about $\psi_{\mathrm{a}}=10^{-4}$ or smaller. To model them, the time evolution of the $\mathrm{m} / \mathrm{n}=2 / 1$ component trigger is taken to be similar to that shown in figure 1 with $1 / \gamma_{\mathrm{f}}=50 \mu \mathrm{s}$ and $\psi_{\mathrm{a}, \max }=10^{-4}$. After $\psi_{\mathrm{a}}=10^{-4}$ is reached, the external field perturbation is kept constant for another $2 \mathrm{~ms}$.

The nonlinear growth of the $\mathrm{m} / \mathrm{n}=2 / 1$ island driven by the external field perturbation described above is shown in figure 10 for different values of plasma rotation frequency, $f_{E}=2 \mathrm{kHz}$ and $10 \mathrm{kHz}$, where $f_{\mathrm{E}}$ is the equilibrium plasma rotation frequency at $\mathrm{q}=2$ surface introduced by the momentum source in equation (3). $f_{\mathrm{E}}>0$ refers to the plasma rotation in the ion diamagnetic drift direction. Here the full set of the four-field equations, equations (1)-(4), are utilized, including the electron inertia. The ratio between the current skin depth, $\mathrm{d}_{\mathrm{e}}=\mathrm{C} / \omega_{\mathrm{pe}}$, and $\rho_{\mathrm{s}}$ equals 0.30 , where $\mathrm{C}$ is the light velocity, and $\omega_{\mathrm{pe}}$ is the local electron plasma frequency. For $\mathrm{f}_{\mathrm{E}}=-\mathrm{f}_{\mathrm{e}}=2 \mathrm{kHz}$, the driven mode frequency $\left(\mathrm{f}=\mathrm{f}_{\mathrm{e}}+\mathrm{f}_{\mathrm{E}}=0\right)$ is the same as that of the trigger's, so that the resulting island is the largest. In this case the seed island width reaches about $0.02 \mathrm{a}$ at $\mathrm{t}=1.1 \times 10^{-4} \tau_{\mathrm{R}}$, which corresponds to the time that a constant $\psi_{\mathrm{a}}=10^{-4}$ has lasted for $2 \mathrm{~ms}$. At this time the $\psi_{2 / 1}$ value at the $\mathrm{q}=2$ surface equals $0.06 \psi_{\mathrm{a}}$. The vertical dashed line shows the time when $\psi_{\alpha}$ reaches $\psi_{a, \max }$. For $\mathrm{f}_{\mathrm{E}}=10 \mathrm{kHz}$, being typical for ASDEX Upgrade discharges with neutral beam injection in the plasma current direction, the island width is only about $0.007 \mathrm{a}$ $(0.35 \mathrm{~cm})$ due to the shielding effect of plasma rotation, since the relative frequency between the trigger and the driven mode is $\mathrm{f}=\mathrm{f}_{*_{\mathrm{e}}}+\mathrm{f}_{\mathrm{E}}=8 \mathrm{kHz}$. For the case $\mathrm{f}_{\mathrm{*}_{\mathrm{e}}}=0$, obtained by taking zero 
equilibrium electron density gradient, the seed island width reaches $0.013 \mathrm{a}$ at $\mathrm{t}=1.1 \times 10^{-4} \tau_{\mathrm{R}}$. The shielding effect is somewhat smaller as $\mathrm{f}=\mathrm{f}_{\mathrm{E}}=2 \mathrm{kHz}$. Before $\psi_{\alpha}$ reaches $\psi_{\mathrm{a} \text {, max }}$, the island growth is about the same for different rotation frequencies. This might be due to a shorter growth time of the trigger, $1 / \gamma_{\mathrm{f}}=50 \mu \mathrm{s}$, compared to the mode rotation period $(125 \mu \mathrm{s}$ for a relative rotation frequency $8 \mathrm{kHz}$ ). Results obtained from the reduced MHD equations are also shown in figure 10 for $\mathrm{f}_{\mathrm{E}}=5 \mathrm{kHz}$. In this case the island width is much smaller than the one obtained from twofluid equations.

It is seen that the seed island generated by an (simulated) ELM is small for typical ASDEX Upgrade parameters when taking into account the frequency difference between the mode and the trigger. The island width is much smaller than the poloidal ion gyroradius, which is about a few centimetres for ASDEX-Upgrade. This might explain why $m / n=2 / 1 \mathrm{NTMs}$ triggered by ELMs are rarely seen in ASDEX Upgrade experiments.

To look into the effect of electron inertia, the nonlinear growth of the $m / n=2 / 1$ island is shown in figure 11 for two cases, including $\left(\mathrm{m}_{\mathrm{e}}>0\right.$, solid curve $)$ and neglecting $\left(\mathrm{m}_{\mathrm{e}}=0\right.$, dotted curve) the electron inertia, with $\mathrm{f}_{\mathrm{E}}=\mathrm{f}_{*_{\mathrm{e}}}=0$. The island width is about the same as that for $\mathrm{f}_{\mathrm{E}}=-$ $\mathrm{f}_{*_{\mathrm{e}}}=2 \mathrm{kHz}$ shown in figure 10. Electron inertia effect leads to a slightly larger island in this case. The dashed curve is obtained from the reduced MHD equations with $f_{E}=0$. The finite $\rho_{s}$ effect causes the major difference from the reduced MHD equations.

The effect of the trigger's amplitude on the $\mathrm{m} / \mathrm{n}=2 / 1$ island growth, obtained from the fourfield equations, is shown in figure 12 for $\mathrm{f}_{\mathrm{E}}=\mathrm{f}_{*_{\mathrm{e}}}=0$ with $\psi_{\mathrm{a}, \max }=10^{-5}, 2 \times 10^{-5}, 5 \times 10^{-5}, 10^{-4}$, and $2 \times 10^{-4}$. The other parameters are the same as those for figure 10 . The island width at $\mathrm{t}=1.1 \times 10^{-}$ ${ }^{4} \tau_{\mathrm{R}}$, the time that a constant $\psi_{\mathrm{a}}$ has lasted for $2 \mathrm{~ms}$ for $\psi_{\mathrm{a}, \max }=10^{-4}$, is not linearly proportional to the value of $\psi_{\mathrm{a}, \max }$. With increasing $\psi_{\mathrm{a} \text {,max }}$ by 10 times from $10^{-5}$ to $10^{-4}$, the island width is increased by 2.7 times. There is a slight difference in the time when $\psi_{\mathrm{a}}$ reaches $\psi_{\mathrm{a} \text {,max }}$ in figure 
12 for different values of $\psi_{\mathrm{a} \text {,max }}$, but the difference is only $0.64 \times 10^{-5} \tau_{\mathrm{R}}$ between the two cases $\psi_{\mathrm{a}, \max }=10^{-5}$ and $2 \times 10^{-4}$.

The duration of the trigger perturbation is found to be important for the induced seed island width. If we assume here a time trace for a trigger perturbation to be a short pulse of the form

$$
\psi_{\mathrm{a}}=\psi_{\mathrm{a} 0} \exp \left\{\gamma_{\mathrm{f}} \mathrm{t} /\left[1+\left(\mathrm{t} / \mathrm{t}_{0}\right)^{\mathrm{i}}\right]\right\}
$$

then $\psi_{\mathrm{a}}$ grows exponentially for $\left(\mathrm{t} / \mathrm{t}_{0}\right)^{\mathrm{i}}<<1$. In the opposite case it decays to $\psi_{\mathrm{a} 0}=10^{-10} . \psi_{\mathrm{a}}$ has a maximum $\psi_{\mathrm{a}, \max }$ at $\mathrm{t}=\mathrm{t}_{0}(\mathrm{i}-1)^{-1 / \mathrm{i}}$. The time evolution of $\psi_{\mathrm{a}}\left(\times 10^{2}\right)$ is shown in figure 13 by the dashed curve using $1 / \gamma_{\mathrm{f}}=50 \mu \mathrm{s}, \psi_{\mathrm{a}, \max }=10^{-4}$ and $\mathrm{i}=8$. The corresponding growth of the $\mathrm{m} / \mathrm{n}=2 / 1$ island is shown by the solid curve for $\mathrm{f}_{\mathrm{E}}=\mathrm{f}_{\mathrm{*}_{\mathrm{e}}}=0$, obtained by neglecting the electron inertia. The induced island width is about three times smaller than that shown in figure 11 for this case of a short lasting trigger perturbation. Inclusion of the electron inertia is found to only slightly increase the island width, similar to the case given in figure 11. The island width obtained from the reduced MHD equations is also shown by the dotted curve. The island decays slowly after the trigger disappears because of the low plasma resistivity.

Assuming parameters relevant for a fusion reactor, $B_{0 t}=6 T, a=2 m, R=6 m, \mu=0.2 \mathrm{~m}^{2} / \mathrm{s}$, $\mathrm{D}_{\perp}=\mu / 5, \mathrm{~T}_{\mathrm{e}}=10 \mathrm{keV}$ and $\mathrm{n}_{\mathrm{e}}=10^{20} \mathrm{~m}^{-3}$ at the resonant surface, one has $\mathrm{S}_{\mathrm{s}}=1.94 \times 10^{10}, \Omega=1.8 \times 10^{6}$ $C_{s}=2.05 \times 10^{9}\left(a / \tau_{R}\right), d_{1}=3.14 \times 10^{8}, \tau_{R}=4202 s, \mu=210\left(a^{2} / \tau_{R}\right), v_{e i}=6.59 \times 10^{3} / s$, and $\rho_{s}=0.0012 a . \quad$ In figure 14 the growth of the $m / n=2 / 1$ island, obtained from the two-fluid equations, is shown for $\mathrm{f}_{\mathrm{E}}=\mathrm{f}_{*_{\mathrm{e}}}=0$ (solid curve). For this case the same trigger perturbation as that for figure 10 has been applied. The induced seed island width reaches about $0.003 \mathrm{a}$ at $\mathrm{t}=6.4 \times 10^{-7} \tau_{\mathrm{R}}$, which corresponds to the time that the trigger perturbation $\psi_{\mathrm{a}}=10^{-4}$ has lasted for $2 \mathrm{~ms}$. The vertical dashed line shows the time when $\psi_{\mathrm{a}}$ reaches its maximal value $\psi_{\mathrm{a}, \max }=10^{-4}$. The dotted curve is obtained from the reduced MHD equations. When a finite frequency difference between the driven mode and the trigger is taken into account, the driven island is found to be even smaller, 
as expected. In the linear phase the driven modes are found to be the kink-like type for both cases. The even smaller induced island compared to the ASDEX-Upgrade case considered above is due to the reduced values of plasma resistivity and $\rho_{\mathrm{s}}$ in this case. This is consistent with corresponding analytical results based on reduced MHD equations [27].

\section{Discussion and summary}

The theories for spontaneously growing tearing modes have been well established in the cold-ion limit and are characterized into to the collisional, semi-collisional and collisionless tearing mode in the linear phase for different plasma parameters $[41,44]$. The key difference between a spontaneously growing mode and a driven one is in the linear growth rate, being determined by the plasma parameters and the equilibrium current density profile for spontaneously growing modes but by the trigger's growth rate for driven modes, as seen from figure 2. If there is no additional drive, the ideal kink modes with $m>1$ are known to be stable if their resonant surfaces are well inside the plasma. Sufficiently fast growing magnetic perturbations due to ELMs or other instabilities can however drive the kink-like mode to grow. This mode only exists during the fast growing period of the trigger and becomes a tearing mode later when the trigger's growth slows down. In this case a trigger having a larger amplitude and lasting for a longer time period after $\psi_{\mathrm{a}}=\psi_{\mathrm{a}, \max }$ is reached leads to a larger seed island, since there is little magnetic reconnection for the driven kink mode during the fast growing phase of the trigger, as seen from figures 7 and 14. This is different from the driven tearing mode shown in figure 3, for which the island significantly grows during the growing phase of the trigger.

The theory of small island physics is quite complex [13-24]. A small island is predicted to be stabilized by several effects, e.g., the ion polarization current, when its width is smaller than a marginal width being close to the poloidal ion gyroradius. Experimentally it is very difficult to directly measure the width of small islands. If the vacuum assumption is made for obtaining 
the magnetic field perturbation at the resonant surface from that at magnetic probes for calculating the island width, the field perturbations at these two places are of the same order of magnitude for $m=2$. Our numerical results show that they differ by one or two orders of magnitude during the fast growing phase of the trigger, indicating the strong plasma shielding in this phase.

It should be mentioned that electron temperature perturbations are neglected here. The small island driven by the electron temperature gradient and its transition into NTMs can be found in separate papers $[25,26]$. Calculations of the seed island formation triggered by sawtooth crash will be carried out in the future.

In summary, the growth of seed magnetic islands driven by magnetic triggers is studied using the four-field equations. The trigger is modelled by externally applied RMPs. It is found that:

(1) In the linear phase a slowly growing trigger drives a tearing mode, while a fast one drives a kink-like mode. The kink-like mode becomes a tearing mode later when the trigger's growth slows down.

(2) Comparing with the results obtained from reduced MHD equations, finite $\rho_{\text {s }}$ effects extend the driven tearing mode regime to a wider range of plasma parameters and lead to larger seed islands in the nonlinear phase. Electron inertia effect also increases the seed island width.

(3) Plasma rotation or electron diamagnetic drift, when increasing the relative rotation between the trigger and the driven mode, decrease the seed island width as expected. For typical ASDEX Upgrade parameters and a frequency difference $8 \mathrm{kHz}$ between the trigger and the driven mode, the generated $\mathrm{m} / \mathrm{n}=2 / 1$ seed island is about $0.007 \mathrm{a}(0.35 \mathrm{~cm})$ for $\psi_{\mathrm{a}, \max }=10^{-4}$. When neglecting the frequency difference, the seed island width is about $0.02 \mathrm{a}(1 \mathrm{~cm})$, being close to the poloidal ion gyroradius.

(4) For a fusion reactor like ITER, if one neglects the frequency difference between the trigger 
and the driven mode and assumes that $\psi_{a, \max }=10^{-4}$ lasts for $2 \mathrm{~ms}$, the generated $\mathrm{m} / \mathrm{n}=2 / 1$ seed island is about $0.003 \mathrm{a}(0.6 \mathrm{~cm})$, being much smaller than the poloidal ion gyroradius.

Acknowledgement: We are grateful to helpful discussions with Dr. A. Gude. One of the authors (K.L.) would like to acknowledge the support by the Austrian Science Fund (FWF) under grant No. P19901. 


\section{References}

[1] Z. Chang, J. D. Callen, E. D. Fredrickson et al., Phys. Rev. Letts, 74, 4663 (1995).

[2] H. Zohm, G. Gantenbein, A. Gude, S. Günter et al., Phys. Plasmas 8, 2009 (2001).

[3] S. Günter, A. Gude, M. Maraschek, S. Sesnic et al., Phys. Rev. Letters, 87, 275001 (2001).

[4] R. J. La Haye, L. L. Lao, E. J. Strait, and T. S. Taylor, Nucl. Fusion 37, 397 (1997).

[5] O. Sauter, R.J. La Haye, Z. Chang, D.A. Gates et al., Phys. Plasmas 4, 1654 (1997).

[6] S. Günter, A. Gude, M. Maraschek, et al., Nucl. Fusion 38, 1431 (1998).

[7] A. Gude, S. Günter, S. Sesnic, ASDEX Upgrade Team, Nucl. Fusion 39, 127 (1999).

[8] R.J. Buttery, R. J. La Haye, P. Gohil, G.L. Jackson, et al., Phys. Plasmas 15, 056115 (2008).

[9] R. J. La Haye and O. Sauter, Nucl. Fusion 38, 987 (1998)

[10] R. J. La Haye, P.A. Politzer and D.P. Brenna, Nucl. Fusion 48, 015005 (2008).

[11] R. J. La Haye, D.P. Brenna, R.J. Buttery, Phys. Plasmas 17, 056110 (2010).

[12] E. D. Fredrickson, Phys. Plasmas 9, 548 (2002).

[13] J.F. Drake, T.M. Antonsen, A.B. Hassam, and N.T. Gladd, Phys. Fluids 26, 2509 (1983).

[14] Bruce D. Scott, and A. B. Hassam, Phys. Fluids 30, 90 (1987).

[15] A. I. Smolyakov, Plasma Phys. Control. Fusion, 35, 657 (1993).

[16] H. R. Wilson, J.W. Connor, R.J. Hastie, and C. C. Hegna, Phys. Plasmas 3, 248 (1996).

[17] F. L. Waelbroeck and R. Fitzpatrick, Phys. Rev. Letts, 78, 1703 (1997).

[18] F.L.Waelbroeck, J.W. Connor, and H. R. Wilson, Phys. Rev. Letters, 87, 215003 (2001).

[19] J.W. Connor, F. L. Waelbroeck, and H. R. Wilson, Phys. Plasmas 8, 2835 (2001).

[20] A.I. Smolyakov, H.R. Wilson, M. Ottaviani and F. Porcelli, Plasma Phys. Control. Fusion, 46, L1 (2004).

[21] R. Fitzpatrick, F. L. Waelbroeck, and F. Militello, Phys. Plasmas 13, 122507 (2006).

[22] F.L.Waelbroeck, Plasma Phys. Control. Fusion, 49, 905 (2007).

[23] A. H. Glasser, J. M. Greene, and J. L. Johnson, Phys. Fluids 19, 567 (1976). 
[24] H. Lütjens, J-F Luciani and X. F. Garbet, Phys. Plasmas 8, 4267 (2001).

[25] Q. Yu, Nucl. Fusion 50, 025014 (2010).

[26] Q. Yu, S. Günter, B. D. Scott, Phys. Plasmas 10, 797 (2003).

[27] C. C. Hegna and J. D. Callen, and R. J. La Haye, Phys. Plasmas 6, 130 (1999).

[28] R. Fitzpatrick, Nucl. Fusion 33, 1049 (1993).

[29] H.R. Koslowski, Y. Liang A. Krämer-Flecken et al., Nucl. Fusion 46, L1 (2006).

[30] Q. Yu, S. Günter, Y. Kikuchi, and K. H. Finken, Nuclear Fusion 48, 024007 (2008).

[31] F. L. Waelbroeck, Phys. Plasmas 10, 4040 (2003).

[32] R. Fitzpatrick and T.C. Hender, Phys. Fluids B3, 644 (1991).

[33] Q. Yu, S. Günter and K.H. Finken, Phys. Plasmas 16, 042301 (2009).

[34] Q. Yu and S. Günter, Nucl. Fusion 49, 062001 (2009).

[35] T. S. Hahm and R. M. Kulsrud, Phys. Fluids 28, 2412 (1985).

[36] X. Wang and A. Bhattacharjee, Phys. Plasmas 4, 748 (1997).

[37] Z. W. Ma, X. Wang, and A. Bhattacharjee, Phys. Plasmas 3, 2427 (1996).

[38] S. Schmidt, S. Günter and K. Lackner, Phys. Plasmas 16, 072302 (2009).

[39] A. Y. Aydemir, Phys. Plasmas 12, 080706 (2005).

[40] R. G. Kleva, J. F. Drake, and F. L. Waelbroeck, Phys. Plasmas 2, 1 (1995).

[41] J. F. Drake and Y. C. Lee, Phys. Fluids 20, 1341 (1977).

[42] B. Coppi, R. Galvão, R. Pellat, M. Rosenbluth, and P. Rutherford, Sov. J. Plasma Phys. 2, $533(1976)$

[43] E. Wolfrum, A. Burckhart, R. Fischer et al., Plasma Phys. Control. Fusion 51, 124057 (2009).

[44] H. P. Furth, J. Killeen, and M. N. Rosenbluth, Phys. Fluids 6, 456 (1963).

[45] P. H. Rutherford, Phys. Fluids 16, 1903 (1973).

[46] P. L. Pritchett, Y. C. Lee and J. F. Frake, Phys. Fluids 23, 1368 (1980). 


\section{CAPTION}

Figure 1 Time evolution of the external perturbation $\psi_{\mathrm{a}}$ with $\mathrm{m} / \mathrm{n}=2 / 1$ (dashed curve), which grows exponentially in time with $\gamma_{\mathrm{f}}=2 \times 10^{5} / \tau_{\mathrm{R}}$ until $\psi_{\mathrm{a}}=\psi_{\mathrm{a}, \max }=10^{-4}$, and afterwards it is kept constant. The solid curve is the induced $\mathrm{m} / \mathrm{n}=2 / 1$ component of the normalized helical flux, $\psi_{\mathrm{s}}=\psi_{2 / 1} / \mathrm{aB} \mathrm{B}_{0 \mathrm{t}}$, at the $\mathrm{q}=2$ surface, being much smaller than that at the plasma edge. The dotted curve shows the normalized helical flux at the $\mathrm{q}=2$ surface using vacuum assumption.

Figure 2 Corresponding to figure 1, the solid curve shows the time evolution of the normalized growth rate of the driven mode, $\gamma \tau_{R}$, calculated from $\left(\mathrm{d} \psi_{2 / 1} / \mathrm{dt}\right) / \psi_{2 / 1}$ at $\mathrm{q}=2$ surface. The dashed curve is the same as the solid one except for a smaller $\psi_{\mathrm{a} 0}\left(=10^{-14}\right)$ and $\psi_{\mathrm{s}}$ at $\mathrm{t}=0$. Three different phases are seen: (A) Initial phase: $\gamma$ approaches $\gamma_{\mathrm{f}}$ (B) Linear phase: $\gamma$ is the same as $\gamma_{\mathrm{f}} .(C)$ Non-constant growth rate phase: $\gamma$ deviates from $\gamma_{\mathrm{f}}$ due to the deviation from the exponential growth of the trigger, beginning when $\psi_{\mathrm{a}}$ reaches a constant. For a sufficiently large $\gamma_{\mathrm{f}}$ and $\psi_{\mathrm{a}, \max }$, however, this phase begins earlier before $\psi_{\mathrm{a}}$ reaches a constant due to nonlinear effect.

Figure 3 Growth of the $m / n=2 / 1$ island corresponding to figure 1 , where $r_{+}$is the island edge at $r>r_{s}$ at the helical angle passing through the island's o-point, and $r_{\text {. }}$ is that at $r<r_{s}$. The island width reaches about $0.01 \mathrm{a}$ at the end of the linear phase, as indicated by the vertical dashed line.

Figure 4 (a) Local radial profiles of the normalized $\psi_{2 / 1}, \psi_{2 / 1} / \mathrm{aB}_{0 \mathrm{t}}$, in the linear phase with $\gamma_{\mathrm{f}}=10^{6} / \tau_{\mathrm{R}}$. For a larger $\mathrm{S}$ (smaller $\tau_{\mathrm{A}}$, as the $\tau_{\mathrm{R}}$ value is fixed in calculations), $\psi_{2 / 1}$ is larger than zero across the resonant surface at $\mathrm{r}=0.628 \mathrm{a}$, being a tearing mode type. With decreasing $\mathrm{S}$ (increasing $\tau_{\mathrm{A}}$ ), $\psi_{2 / 1}$ is positive for $\mathrm{r}>\mathrm{r}_{\mathrm{s}}$ but negative for $\mathrm{r}<\mathrm{r}_{\mathrm{s}}$, showing the feature of an ideal mode. (b) Corresponding radial profiles of the normalized stream function, $\phi_{2 / 1} / \mathrm{a}^{2} \tau_{\mathrm{R}}$. It changes 
sign across the resonant surface for a large $\mathrm{S}$ but takes the same sign for a smaller $\mathrm{S}$.

Figure 5 Boundary between the driven tearing and driven kink mode in the $S$ and $\gamma_{\mathrm{f}} \tau_{\mathrm{A}}$ plane for $\rho_{\mathrm{s}}=0.02 \mathrm{a}$ (solid curve) and 0 (dashed curve) in the linear phase. For $\rho_{\mathrm{s}}=0$ the driven kink mode regime exists for $\tau_{\mathrm{A}} \gamma_{\mathrm{f}}>\mathrm{cS}_{\mathrm{s}}^{-1 / 3}$, as predicted by (A18) in the Appendix, where $\mathrm{c}=1.02, \mathrm{c}_{1}=\mathrm{c} /$ $\left(\mathrm{k}_{\|}{ }^{2}\right)^{2 / 3}=0.88$, and $\mathrm{S}_{\mathrm{s}}=\mathrm{S} \eta_{0} / \eta_{\mathrm{s}}$. The finite $\rho_{\mathrm{s}}$ effect extends the tearing mode regime to large $\gamma_{\mathrm{f}}$ values.

Figure 6 Local radial profiles of the $m / n=2 / 1$ component of the normalized current density in the linear phase for $\rho_{\mathrm{s}}=0,0.0067 \mathrm{a}$ and $0.02 \mathrm{a}$ with $\gamma_{\mathrm{f}}=10^{6} / \tau_{\mathrm{R}}(\mathrm{a})$, which is more peaked around the resonant surface for a larger $\rho_{\mathrm{s}}$. Corresponding radial profiles of the $2 / 1$ component of plasma vorticity, $\mathrm{U}_{2 / 1}$, and stream function, $\phi_{2 / 1}$, are shown in (b) and (c), respectively.

Figure 7 Nonlinear growth of $\mathrm{m} / \mathrm{n}=2 / 1$ island for $\gamma_{\mathrm{f}} \tau_{\mathrm{R}}=10^{7}$ with $\rho_{\mathrm{s}}=0.02 \mathrm{a}$ (solid curve) and $\rho_{\mathrm{s}}=0$ (dotted). There is essentially no magnetic reconnection in the linear phase due to the large $\gamma_{\mathrm{f}}$, as the driven modes are of the kink-like type for both cases. The transition from the kink-like to tearing mode begins when $\psi_{\mathrm{a}}$ reaches a constant, $\psi_{\mathrm{a}, \max }=10^{-4}$, as indicated by the vertical dashed line, and the island width grows to about $0.01 \mathrm{a}$ in a time scale $10^{-6} \tau_{\mathrm{R}}$, being faster for $\rho_{\mathrm{s}}=0.02 \mathrm{a}$ than that for $\rho_{\mathrm{s}}=0$. The outwards shift of the island edges is due to the $\mathrm{m} / \mathrm{n}=0 / 0$ component of plasma current density perturbation.

Figure 8 Nonlinear growth of normalized $\mathrm{m} / \mathrm{n}=2 / 1$ island width, W/a, with $\gamma_{\mathrm{f}} \tau_{\mathrm{R}}=10^{7}$ for $\rho_{\mathrm{s}}=0.02 \mathrm{a}, 0.01 \mathrm{a}, 0.0045 \mathrm{a}$ and 0 . The vertical dashed line shows the time when $\psi_{\mathrm{a}}$ reaches $\psi_{\mathrm{a}, \max }=10^{-4}$. The island quickly grows from that time and grows faster for a larger $\rho_{\mathrm{s}}$.

Figure 9 Time evolution of the island width for spontaneously growing tearing modes with $\rho_{\mathrm{s}} / \mathrm{a}=0.02,0.002,0.00067$ and 0 . The faster island growth for a larger $\rho_{\mathrm{s}}$ is due to a larger linear growth rate, being different from the driven mode which linear growth rate is determined by that of the trigger as seen from figure 2. 
Figure 10 Nonlinear growth of the $m / n=2 / 1$ island for $f_{E}=2 k H z$ and $10 \mathrm{kHz}$. For $f_{E}=-f_{*}=2 k H z$, the mode frequency $\left(\mathrm{f}=\mathrm{f}_{\mathrm{e}}+\mathrm{f}_{\mathrm{E}}=0\right)$ is the same as that of the trigger's, so that the resulting island is the largest. The seed island width reaches near $0.02 \mathrm{a}$ at $\mathrm{t}=1.1 \times 10^{-4} \tau_{\mathrm{R}}$, corresponding to the time that $\psi_{\mathrm{a}}=10^{-4}$ has lasted for $2 \mathrm{~ms}$. The vertical dashed line shows the time when $\psi_{\alpha}$ reaches $\psi_{\mathrm{a} \text {,max }}$. For $\mathrm{f}_{\mathrm{E}}=10 \mathrm{kHz}$, the island width is about $0.007 \mathrm{a}(0.35 \mathrm{~cm})$ due to the shielding effect of plasma rotation. For the case $\mathrm{f}_{\mathrm{*}_{\mathrm{e}}}=0$, the seed island width reaches $0.013 \mathrm{a}$ at $\mathrm{t}=1.1 \times 10^{-4} \tau_{\mathrm{R}}$ due to $\mathrm{f}=\mathrm{f}_{\mathrm{E}}=2 \mathrm{kHz}$. The island width obtained from the reduced MHD equations for $\mathrm{f}_{\mathrm{E}}=5 \mathrm{kHz}$ is much smaller than that obtained from two-fluid equations.

Figure 11 Nonlinear growth of the $m / n=2 / 1$ island obtained from two-fluid equations for two cases: including $\left(\mathrm{m}_{\mathrm{e}}>0\right.$, solid curve $)$ and neglecting $\left(\mathrm{m}_{\mathrm{e}}=0\right.$, dotted $)$ the electron inertia, with $\mathrm{f}_{\mathrm{E}}=\mathrm{f}_{*_{\mathrm{e}}}=0$. Electron inertia effect leads to a slightly larger island in this case. The dashed curve is obtained from the reduced MHD equations with $\mathrm{f}_{\mathrm{E}}=0$. The vertical dashed line shows the time when $\psi_{\mathrm{a}}$ reaches $\psi_{\mathrm{a}, \max }=10^{-4}$.

Figure 12 The effect of the trigger's amplitude on the $m / n=2 / 1$ island growth for $f_{E}=f_{*_{e}}=0$ with $\psi_{\mathrm{a}, \max }=10^{-5}, 2 \times 10^{-5}, 5 \times 10^{-5}, 10^{-4}$, and $2 \times 10^{-4}$. The other parameters are the same as those for figure 10. With increasing $\psi_{\mathrm{a} \text {,max }}$ by 10 times from $10^{-5}$ to $10^{-4}$, the island width at $\mathrm{t}=1.1 \times 10^{-4} \tau_{\mathrm{R}}$ (a constant $\psi_{\mathrm{a}}$ has lasted for $2 \mathrm{~ms}$ for $\psi_{\mathrm{a}, \max }=10^{-4}$ ) is increased by 2.7 times. There is a slight difference in the time when $\psi_{\mathrm{a}}$ reaches $\psi_{\mathrm{a}, \max }$ for different values of $\psi_{\mathrm{a}, \mathrm{max}}$,

Figure 13 Nonlinear growth of the $\mathrm{m} / \mathrm{n}=2 / 1$ island driven by the $\psi_{\mathrm{a}}$ given by equation (10) with $\mathrm{f}_{\mathrm{E}}=\mathrm{f}_{*_{\mathrm{e}}}=0$ and $\mathrm{m}_{\mathrm{e}}=0$ (solid curve). The island width is much smaller than that shown in figure 11. The waveform of $\psi_{\mathrm{a}}\left(\times 10^{2}\right)$ is shown by the dashed curve, with $1 / \gamma_{\mathrm{f}}=50 \mu \mathrm{s}$ and $\psi_{\mathrm{a}, \max }=10^{-4}$. The island width obtained from the reduced MHD equation is shown by the dotted curve. The island decays slowly after the trigger disappears.

Figure 14 Nonlinear growth of the $\mathrm{m} / \mathrm{n}=2 / 1$ island obtained from two-fluid equations for 
$\mathrm{f}_{\mathrm{E}}=\mathrm{f}_{\mathrm{e}}=0$ (solid curve) and reactor relevant parameters. The same trigger as that for figure 10 is utilized. The induced seed island width reaches about $0.003 \mathrm{a}$ at $\mathrm{t}=6.4 \times 10^{-7} \tau_{\mathrm{R}}$, which corresponds to the time that $\psi_{\mathrm{a}}=10^{-4}$ has lasted for $2 \mathrm{~ms}$. The vertical dashed line shows the time when $\psi_{\mathrm{a}}$ reaches $\psi_{\mathrm{a}, \max }=10^{-4}$. The dotted curve is obtained from the reduced MHD equations. 


\section{Appendix Heuristic linear analysis from reduced MHD equations}

In reference [35] by Hahm and Kulsrud, most analysis is given for the case that a trigger of a constant amplitude is added as a boundary condition at the beginning in a time scale being shorter than any value of $\tau_{\mathrm{A}}^{\mathrm{S}} \tau_{\mathrm{R}}{ }^{1-\mathrm{s}}$, where $0<\mathrm{S}<1$. In this case the reconnected flux is calculated at different time phases, and it is found that the magnetic reconnection occurs on the time scale about the linear growth time of the resistive tearing mode if the trigger's amplitude is sufficiently small. Hahm and Kulsrud have also considered another case that the trigger grows exponentially in time, and the corresponding reconnected flux is found to grow exponentially too, having the same time dependence $\exp \left(\mathrm{s}_{0} \mathrm{t}\right)$ as that of the trigger (see Eqs. (46) and (47) of reference 35), while the amount of the reconnected flux at a given time is affected by the trigger's growth rate, $\tau_{\mathrm{A}}$ and $\tau_{\mathrm{R}}$.

It will be shown in this Appendix that, if the trigger grows exponentially in time and starts from a sufficiently small amplitude, in the linear phase a slowly growing trigger drives a tearing mode, while a fast one drives a kink-like mode, being in agreement with numerical results shown in Section 3.

When the trigger's amplitude is sufficiently small, the linear approximation is valid, and Ohm's law and the equation of motion can be simplified to

$$
\begin{aligned}
& \gamma_{R} \psi_{1}-i k_{\|} \phi_{1}=-\eta j_{1}, \\
& \gamma_{R} \nabla_{\perp}^{2} \phi_{1}=S^{2} i\left(k_{\|} j_{1}+m \psi_{1} j_{0}{ }^{\prime} / r\right),
\end{aligned}
$$

where the perturbed quantities, denoted by the subscript 1 , are assumed to be proportional to $\exp \left[\gamma_{\mathrm{R}} \mathrm{t}_{\mathrm{R}}+i(\mathrm{~m} \theta+\mathrm{n} \phi)\right]$, being the same as the trigger given by equation (8). The prime denotes the radial gradient, $\mathrm{k}_{\|}=(\mathrm{n}-\mathrm{m} / \mathrm{q}) / \mathrm{R} \approx(\mathrm{n} / \mathrm{R})\left(\mathrm{q}^{\prime} / \mathrm{q}\right) \mathrm{x}$, and $\mathrm{x}=\left(\mathrm{r}-\mathrm{r}_{\mathrm{s}}\right)$. The plasma viscosity is neglected here. All quantities in (A1) and (A2) are in normalized units, as defined in Section2. Only the normalized growth rate, $\gamma \tau_{\mathrm{R}}$, is explicitly labelled as $\gamma_{\mathrm{R}}$. 
The analysis can be carried out in the inner and outer region separately. In the outer region away from the rational surface at $\mathrm{r}=\mathrm{r}_{\mathrm{s}}$, the ideal plasma approximation is valid,

$$
\gamma_{R} \psi_{1}-i k_{\|} \phi_{1}=0,
$$

and (A2) becomes

$$
\frac{1}{r} \frac{d}{d r}\left(r \frac{d \psi_{1}}{d r}\right)-\frac{m^{2}}{r^{2}} \psi_{1}+m \psi_{1} j_{0}{ }^{\prime} /\left(k_{\|} r\right)=0
$$

for large $S$ values. (A3) and (A4) can be utilized to calculate $\psi_{1}, \phi_{1}$ and $\Delta^{\prime}$ in the outer region, to match to those obtained from the inner region.

In the inner region around $\mathrm{r}_{\mathrm{s}}$, one can assume $\phi_{1}{ }^{\prime} \sim \phi_{1} / \varepsilon$ and $\phi_{1}{ }^{\prime \prime} \sim / \varepsilon^{2}$, where $\varepsilon$ is the singular layer width. Depending on the trigger's growth rate, three different types of solutions are possible:

\section{(A) Driven tearing mode under "constant $\psi$ " approximation}

When the growth rate of the trigger is sufficiently small, one can assume that $\mathrm{j}_{1} \approx-\Delta^{\prime} \psi_{1} / \varepsilon$, and (A1) and (A2) lead to

$$
\begin{aligned}
& \gamma_{R} \psi_{l} \sim i k_{\|}{ }^{\prime} \varepsilon \phi_{l}, \\
& \gamma_{R} \sim \eta_{s} \Delta^{\prime} / \varepsilon, \\
& \gamma_{R} \phi_{l} / \varepsilon^{2} \sim S^{2} i k_{\|}{ }^{\prime} \Delta^{\prime} \psi_{l},
\end{aligned}
$$

where $\eta_{s}$ is the normalized plasma resistivity at $r=r_{s}$. From (A5) - (A7) one finds

$$
\begin{aligned}
& \varepsilon \sim\left[\eta_{s}^{2} \Delta^{\prime}\left(S k_{\|}{ }^{\prime}\right)^{-2}\right]^{1 / 5}, \\
& \gamma_{R} \sim\left[\eta_{s}^{3} \Delta^{\prime 4}\left(S k_{\|}{ }^{\prime}\right)^{2}\right]^{1 / 5} .
\end{aligned}
$$

(A8) and (A9) are the same as those of classical tearing modes under the "constant $\psi$ " approximation [44]. The roles of the trigger are to determine the mode growth rate (being the same as that of the trigger) and the value of $\Delta^{\prime}$ from (A9). In the outer region $\psi_{1}$ can be written in the form 


$$
\psi_{1}=\psi_{i}+\psi_{e}
$$

where $\psi_{\mathrm{i}}$ is bounded in $0<\mathrm{r}<\mathrm{a}$, subject to boundary conditions $\psi_{\mathrm{i}}=0$ at $\mathrm{r}=0$ and $\mathrm{r}=\mathrm{a} . \psi_{\mathrm{e}}$ is due to the trigger, bounded in $r_{s}<r<a$ and subject to the boundary condition $\psi_{e}=0$ at $r=r_{s}$. The value of $\psi_{\mathrm{e}}$ at $\mathrm{r}=\mathrm{a}$ is given by equation (8). Matching the $\Delta^{\prime}$ obtained from the inner and the outer region, one finds

$$
\Delta^{\prime}=\Delta_{l}^{\prime}+\psi_{e s}{ }^{\prime} / \psi_{i s}
$$

where the subscript $\mathrm{s}$ corresponds to taking the value at $\mathrm{r}=\mathrm{r}_{\mathrm{s}} . \quad \Delta_{1}^{\prime}$ is obtained from the $\psi_{\mathrm{i}}$ satisfying (A4) and is the usual tearing mode stability index without the trigger, being determined by the q-profile and mode numbers [44]. The second term on the right hand of (A11) is due to the trigger. In order to satisfy (A11), the mode growth rate has to be the same as that of the trigger. In addition, the reconnected flux, $\psi_{\text {is }}$, is determined by (A9) and (A11), being larger for a smaller $\gamma_{R}$ when excluding its time dependence $\exp \left(\gamma_{R} t\right)$ and keeping other parameters unchanged. A larger $\gamma_{R}$ corresponds to a larger $\left|\Delta^{\prime}\right|$ or shielding current around the resonant surface.

In the nonlinear phase, $\Delta^{\prime} \approx(\mathrm{dW} / \mathrm{dt}) / \eta[45]$, and the island growth is found from (A11) to be

$$
\frac{d W}{\eta d t}=\Delta_{1}^{\prime}+\frac{W_{e}^{2}}{W^{2}}
$$

where $\mathrm{W}$ is the island width, and $\mathrm{W}_{\mathrm{e}}{ }^{2}=16(\mathrm{~m} / \mathrm{r}) \psi_{\mathrm{es}}{ }^{\prime} /\left(\mathrm{k}_{\|}{ }^{\prime} \mathrm{B}_{0 \mathrm{t}}\right)[28,32]$.

\section{(B) Driven tearing mode with "Non-constant $\psi$ "}

It is seen from (A9) that, with increasing value of $\gamma_{\mathrm{R}}, \Delta^{\prime}$ becomes larger, indicating that the "constant $\psi$ " approximation is not valid for a sufficiently large $\gamma_{\mathrm{R}}$. In this case one can assume that $\mathrm{j}_{1} \sim-\psi_{1} / \varepsilon^{2}$, and (A1) and (A2) lead to

$$
\gamma_{R} \sim \eta_{s} / \varepsilon^{2}
$$




$$
\gamma_{R} \phi_{1} / \varepsilon^{2} \sim S^{2} i k_{\|}{ }^{\prime} \psi_{1} / \varepsilon
$$

It is found from (A5), (A13) and (A14) that,

$$
\begin{aligned}
& \varepsilon \sim\left[\eta_{s} /\left(S k_{\|}{ }^{\prime}\right)\right]^{1 / 3} . \\
& \gamma_{R} \sim\left[\eta_{s}\left(S k_{\|}{ }^{\prime}\right)^{2}\right]^{1 / 3} .
\end{aligned}
$$

This case is similar to the $m / n=1 / 1$ internal resistive kink mode and the double tearing mode $[42,46]$.

\section{(C) Driven kink mode}

If the trigger's growth rate is even larger than that given by (A16),

$$
\delta=c_{l}\left[\eta_{s}\left(S k_{\|}{ }^{\prime}\right)^{2}\right]^{1 / 3} / \gamma_{R}<1,
$$

the mode growth time is shorter than the resistive diffusion time across the singular layer, $1 / \gamma<\varepsilon^{2} / \eta_{s}$, where $c_{1}$ is a constant of the order of unity. In this case the driven mode cannot be a tearing mode but becomes a kink-like type. (A17) can be also written in the form

$$
\delta=c S_{s}^{-1 / 3} /\left(\tau_{A} \gamma\right)<1
$$

where $\mathrm{c}=\mathrm{c}_{1}\left(\mathrm{k}_{\|}{ }^{\prime}\right)^{2 / 3}$. In the limit $\delta<<1$, to the lowest order the solutions in the inner region are found from (A1) and (A2) to be

$$
\begin{aligned}
& \phi_{1}=c_{2} \gamma_{R}\left[\frac{\pi}{2}+\tan ^{-1}(x / \varepsilon)\right], \\
& \psi_{1}=c_{2} i k_{\|}{ }^{\prime}\left\{\left[\frac{\pi}{2}+\tan ^{-1}(x / \varepsilon)\right] x+2 \delta_{1}^{3} \varepsilon\right\}, \\
& j_{1}=-2 c_{2} i k_{\|}^{\prime} \frac{1}{\varepsilon\left[1+(x / \varepsilon)^{2}\right]^{2}},
\end{aligned}
$$

where

$$
\varepsilon=\tau_{A} \gamma /\left(k_{\|}{ }^{\prime}\right)=\gamma_{R} /\left(S k_{\|}{ }^{\prime}\right)
$$

is the normalized singular layer width, being larger for a larger $\gamma . c_{2}$ is a constant for matching 
the solution to that in the outer region, and $\delta_{1}$ is the $\delta$ given by (A17) with $\mathrm{c}_{1}=1$. The first term on the right hand side of (A20), being proportional to $\mathrm{x}$, is due to the ideal plasma response to the trigger. The last term in (A20) takes into account the small correction from finite plasma resistivity, being important only in a thin layer around $\mathrm{x}=\mathrm{x}_{0} \approx-4\left(\delta_{1}\right)^{3} \varepsilon / \pi$ where $\psi_{1}$ becomes zero. The location of $\mathrm{x}_{0}$ is slightly inside the rational surface as seen from figure $4 \mathrm{a}$, and it approaches $\mathrm{x}=0$ with increasing $\gamma$. As $\mathrm{x}_{0}<<\varepsilon$, there is little magnetic reconnection. The "pure" ideal kink mode is in the limit $\mathrm{x}_{0}=0$, when plasma resistivity approaches zero or when $\gamma$ approaches infinity for a finite plasma resistivity. 


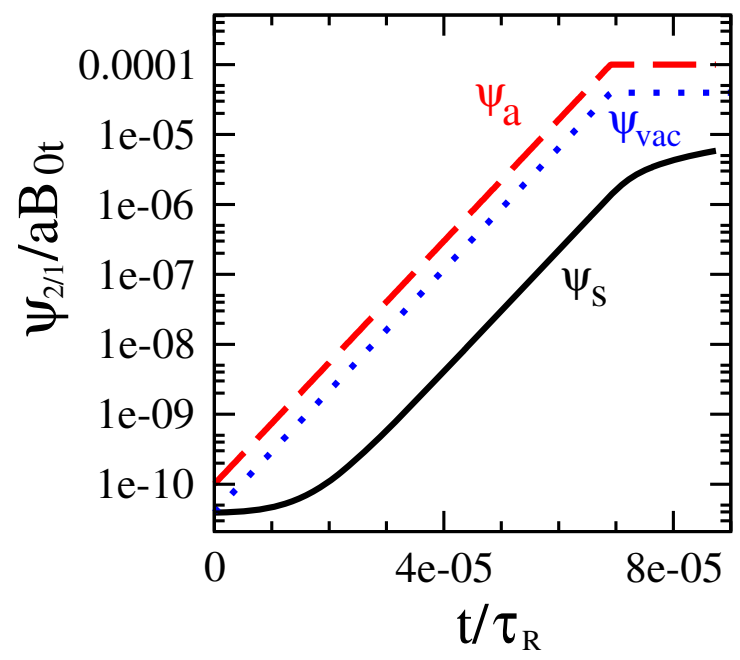

Figure 1 


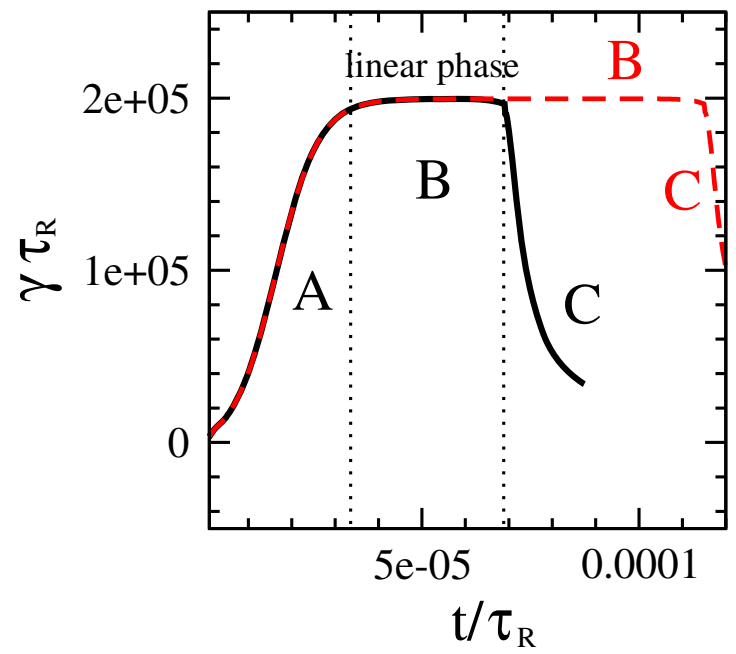

Figure 2 


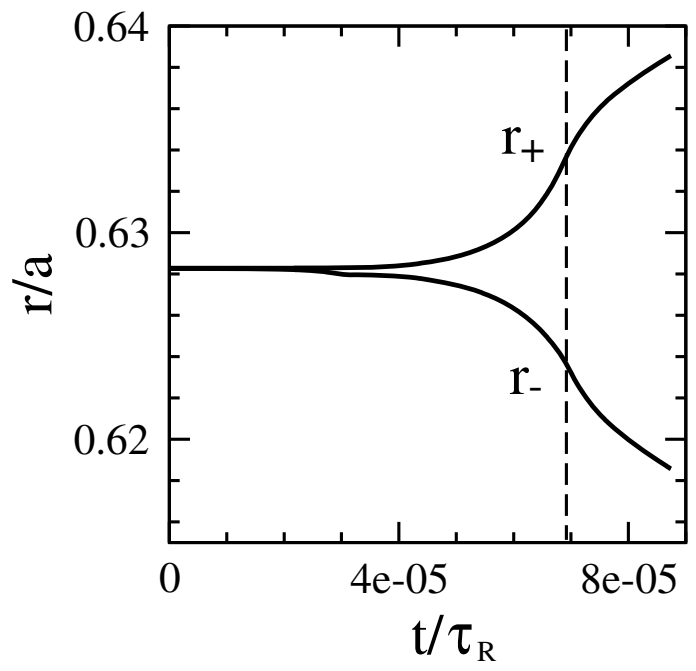

Figure 3 


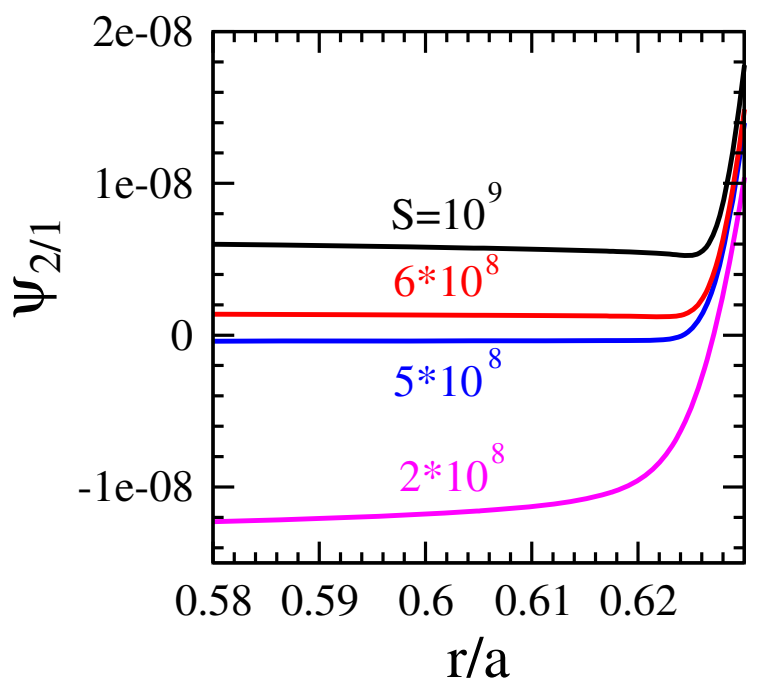

Figure 4a 


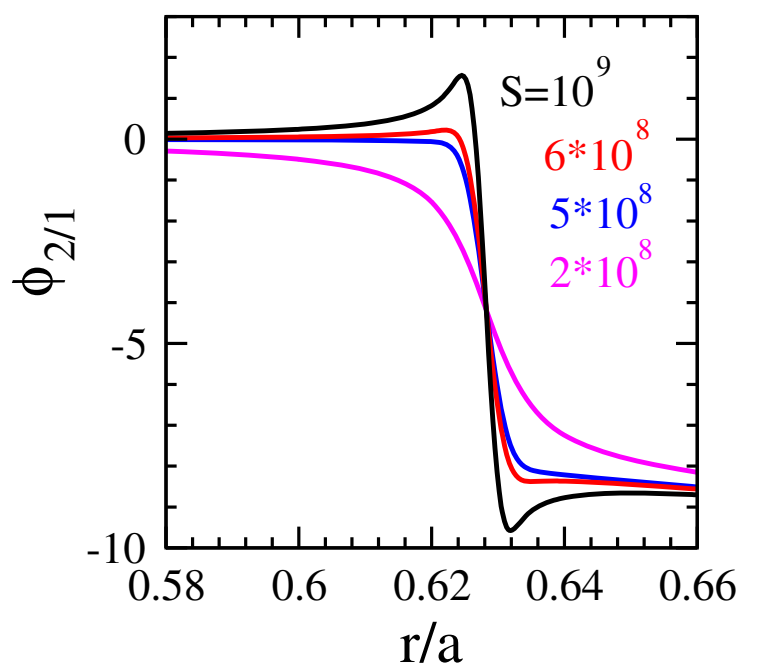

Figure $4 b$ 


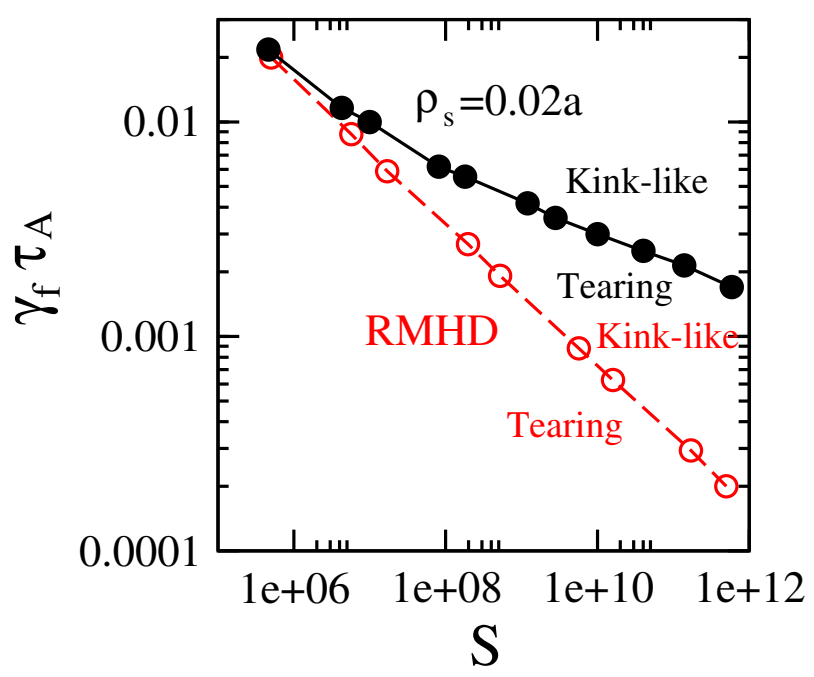

Figure 5 


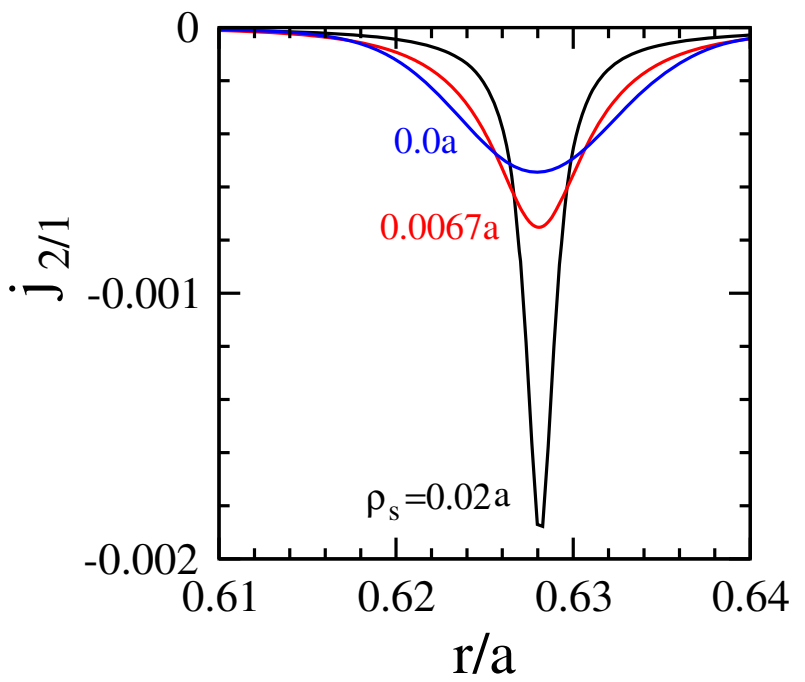

Figure 6a 


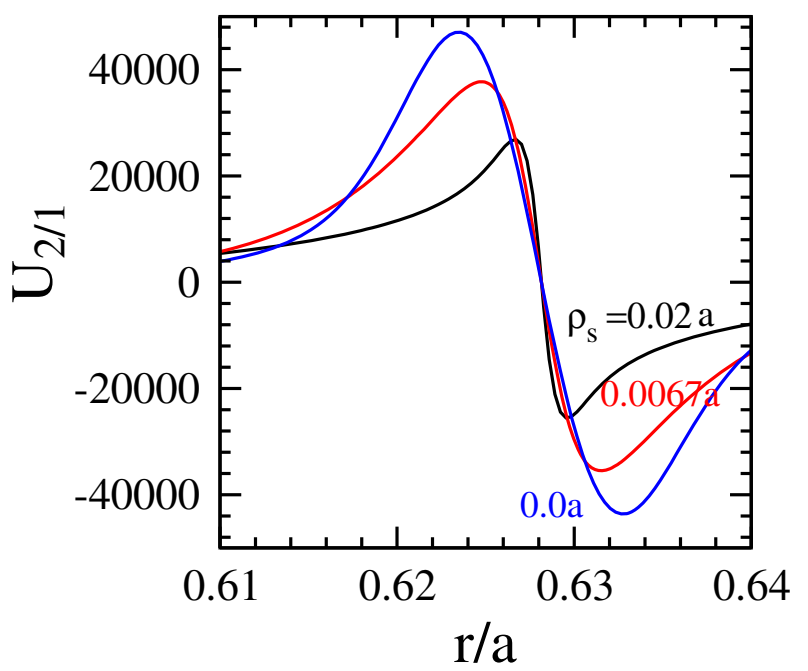

Figure $6 b$ 


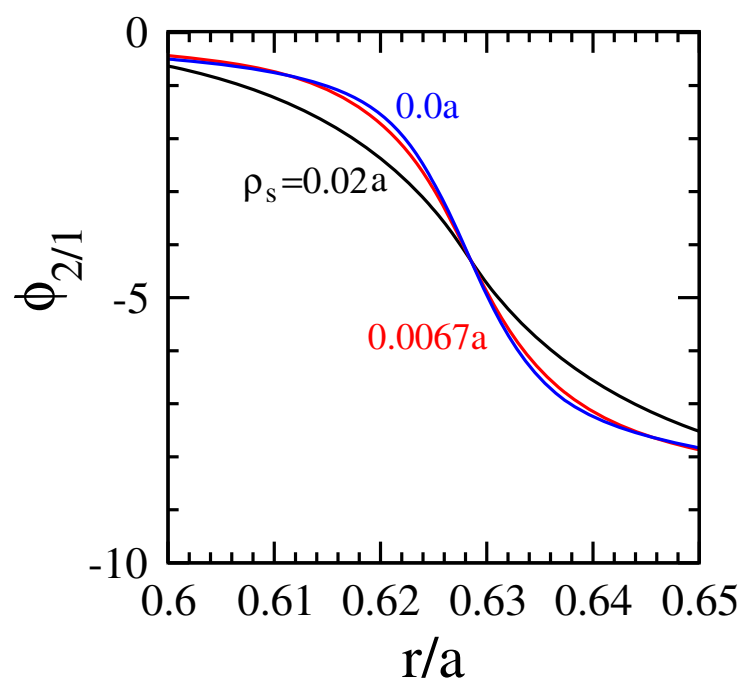

Figure 6c 


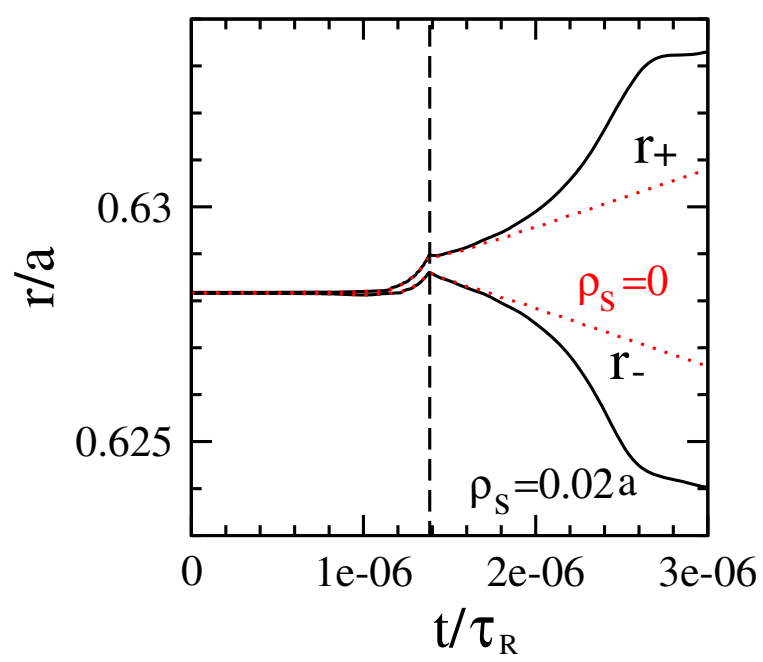

Figure 7 


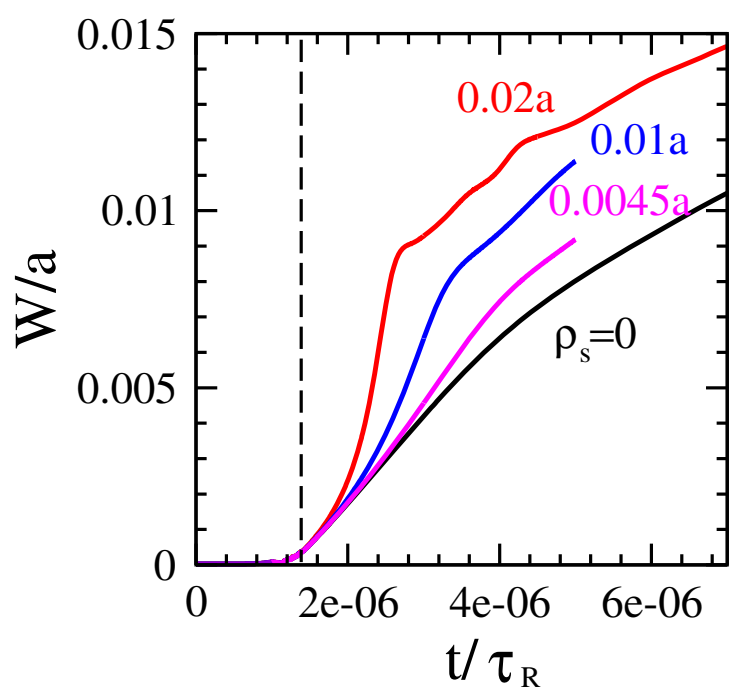

Figure 8 


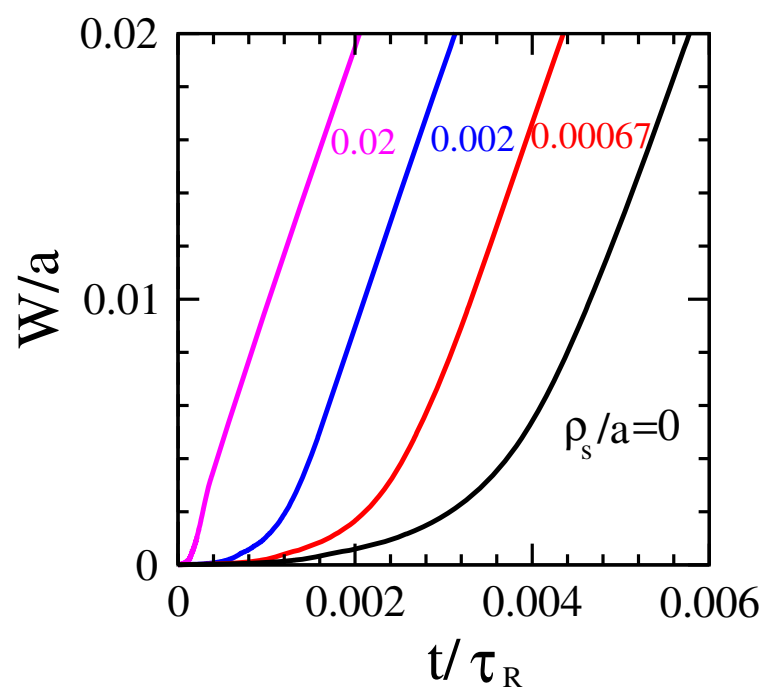

Figure 9 


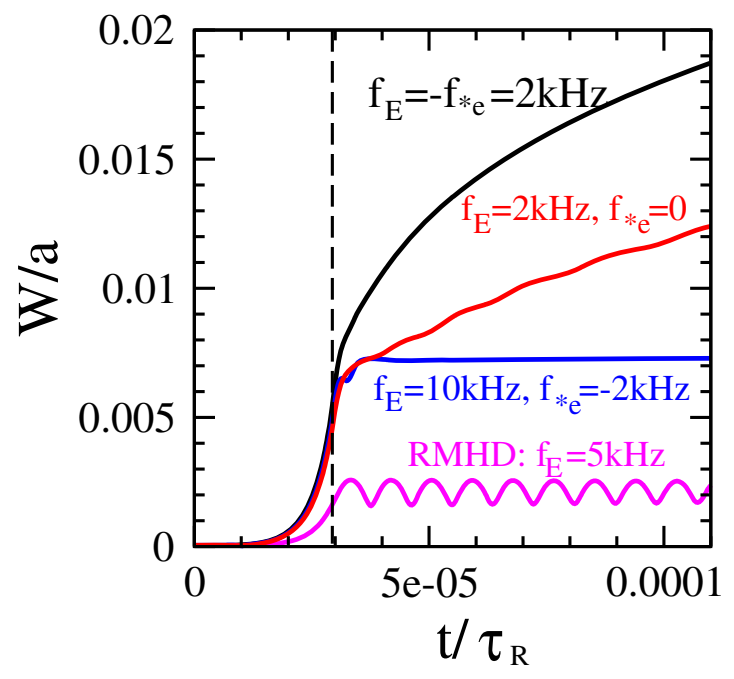

Figure 10 


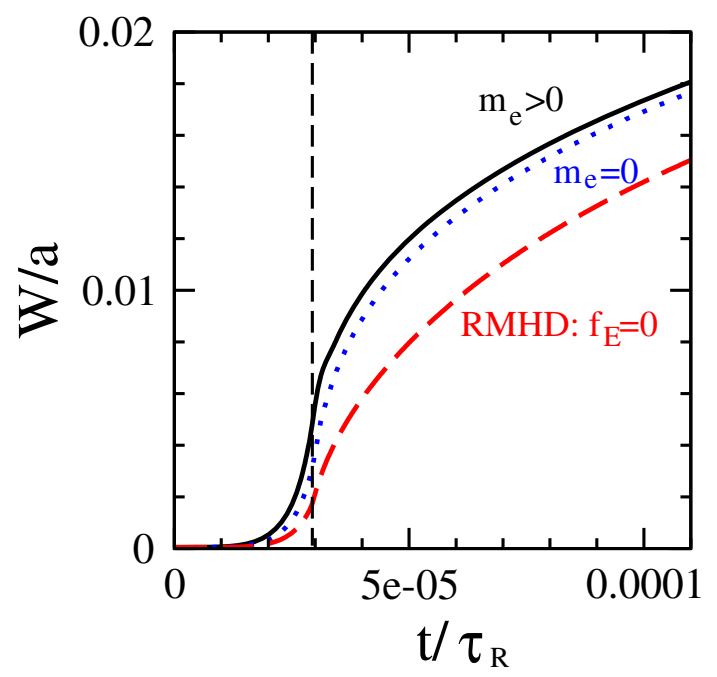

Figure 11 


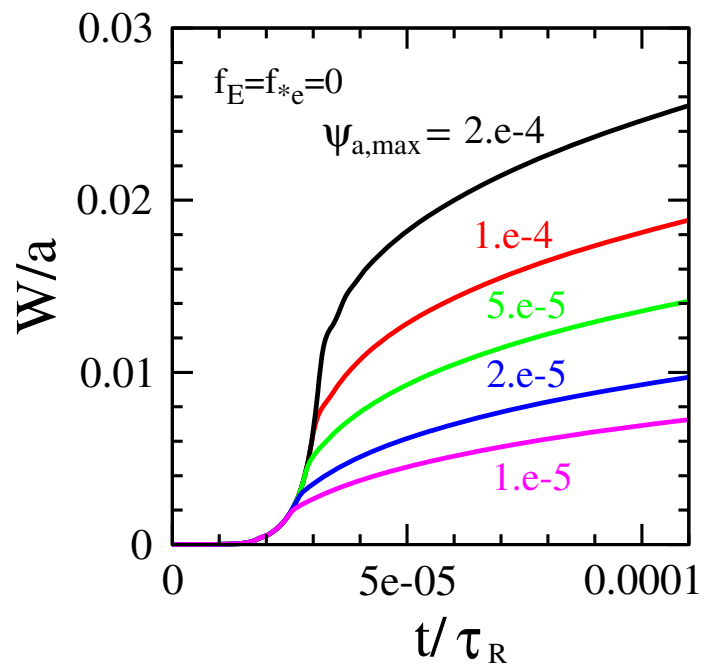

Figure 12 


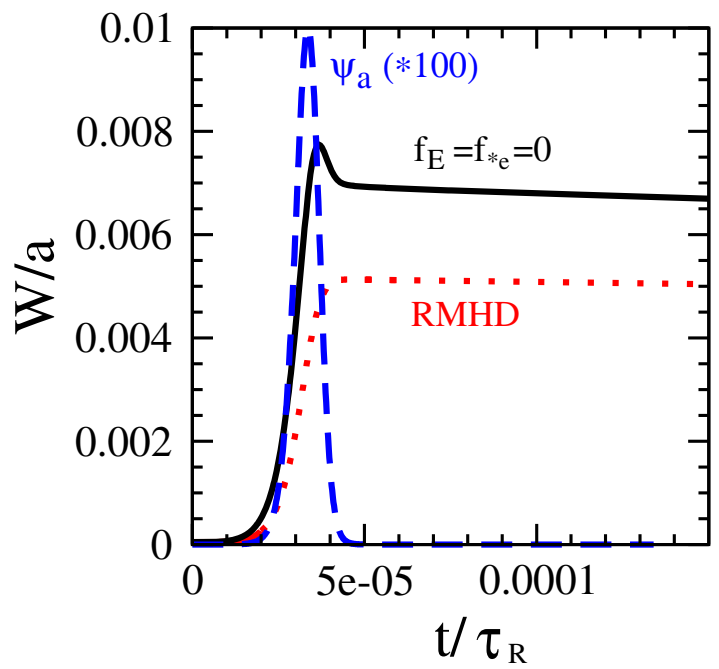

Figure 13 


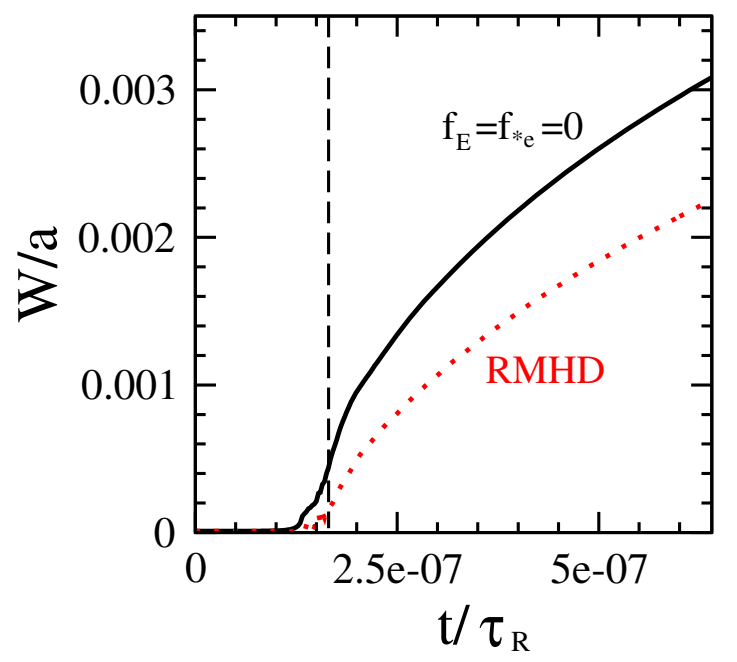

Figure 14 